\title{
DISSIPATION OF ENERGY \\ FOR MAGNETOELASTIC WAVES \\ IN A CONDUCTIVE MEDIUM
}

\author{
BY \\ ELIAS ANDREOU (Department of Mathematics, University of Patras, Greece) \\ AND \\ GEORGE DASSIOS (Univ. of Patras and Institute of Chem. \\ Eng. and High Temperature Chemical Processes, Patras, Greece)
}

\begin{abstract}
We consider the propagation of magnetoelastic waves within a homogeneous and isotropic elastic medium exhibiting finite electric conductivity. An appropriate physical analysis leads to a decoupling of the governing system of equations which in turn effects an irreducible factorization of the ninth-degree characteristic polynomial into a product of first, third, and fifth-degree polynomials. Regular and singular perturbation methods are then used to deduce asymptotic expansions of the characteristic roots which reflect the low and the high frequency dependence of the frequency on the wave number. Dyadic analysis of the spacial spectral equations brings the general solution into its canonical dyadic form. Extensive asymptotic analysis of the quadratic forms that define the kinetic, the strain, the magnetic and the dissipation energy provides the rate of dissipation of these energies as the time variable approaches infinity. The rate of dissipation obtained coincides with the corresponding rate for thermoelastic waves. Therefore, a similarity between the dissipative effects of thermal coupling and that of finite conductivity upon the propagation of elastic waves is established.
\end{abstract}

1. Introduction. Elastic wave propagation in a medium exhibiting electric conductivity is by now an extended branch of continuum mechanics and the reader can consult the fundamental two-volume book by Eringen and Maugin [12] for an almost complete account of the subject. References [16, 20, 21, 22] are also very helpful. Knopoff [18] and Chadwick [6] stated the equations governing the propagation of elastic waves in a solid conductive medium where a homogeneous external magnetic field has been applied and they studied the propagation of plane waves within such a medium. See also the relative work of Banos [1]. The discussion of magnetoelastic waves for parameters that correspond to real earth data can be found in the work of Keilis-Borok and Munin [17]. Dunkin and Eringen [11] published an extensive investigation of the propagation of elastic waves under the influence of a magnetic field and under the influence of an electric field. If thermal effects are also taken into consideration then a much more complicated

Received December 3, 1993.

1991 Mathematics Subject Classification. Primary 35B40, 35Q60, 35Q72, 73D99, 73 R05.

(C) 1997 Brown University 
system is generated and some first useful steps in this direction can be found in the work of Willson [23] and Chander [7]. Lilley and Smylie [19] investigated the extra dissipative effect that is due to the nonuniformity of the external magnetic field, while Boulanger [4] studied the propagation of nonhomogeneous plane waves in a magnetoelastic medium. The geometrical theory of magnetoelastic waves in an inhomogeneous elastic medium that exhibits infinite electric conductivity is given by Bazer [2]. The second author of the present work has discussed some energy theorems connected to partition of energy for magnetoelastic waves in a perfectly conducting medium [9]. Finally, a complete account of the existing literature up to 1968 in magnetoelasticity as well as in magnetothermoelasticity is presented by Paria [22].

In the present work we study the general Cauchy problem, with smooth initial data, within a homogeneous and isotropic elastic medium that has finite electric conductivity. An external homogeneous magnetic field is responsible for the coupling between the elastic and the electromagnetic waves that can propagate within the magnetoelastic medium. Section 2 states the basic equations and incorporates a spacial spectral decomposition into plane wave components. Then the system of equations that governs the time evolution of the disturbances is studied. Its characteristic equation is a ninthdegree polynomial. Section 3 involves a physical analysis of the phenomenon that dictates a corresponding (spherical) decomposition. This leads to a decoupling of the system of equations into three particular systems of the first, the third, and the fifth order. Their characteristic polynomials effect a factorization of the general ninth-degree polynomial into polynomials of degree one, three, and five. This is an important part of our work since, as physical arguments indicate, it is the best possible decoupling. Hence, as is easily observed, the three factors are irreducible real polynomials. In order to study the time evolution of the energies the asymptotic behavior of all nine characteristic roots for small and for large values of the dual spacial variable is needed. This task is easy for the roots of the first and the third-degree polynomials, but it is much harder for the roots of the fifth-degree polynomial. Therefore, a perturbation analysis, regular near the origin and singular near infinity, had to be performed. The results are contained in Sec. 4. In Sec. 5 the reduction of the problem to its standard form [15] is used in order to obtain a contour integral representation of the solution that solves the spectral equation and satisfies the corresponding spectral Cauchy data. The integral is then evaluated using complex residue theory and the solution is transformed into its canonical dyadic form [5], which reveals the physical coupling of the system. In the final Sec. 6 the general energy identity is established and the results of the previous sections are used to obtain the dissipation rates for each one of the kinetic energy, the strain energy, the magnetic energy, and the dissipation function. This is a very laborious program and only the results are provided, since the methods used imitate those for the thermoelastic coupling problem [10]. The rates of dissipation obtained are the same as the corresponding rates for thermoelastic waves [10]. The three energies dissipate at $t^{-(m+3 / 2)}$, where $m$ is a measure of the symmetry of the initial data, while the dissipation function assumes an asymptotic form proportional to $t^{-(m+5 / 2)}$. At each step, the results of this paper recover the corresponding results for perfect conductors [9] as the conductivity of the medium tends to infinity. 
2. Equations of magnetoelastic waves. The linearized equations that describe the coupling between the displacement and the electromagnetic field in a homogeneous and isotropic medium characterized by the Lamé constants $\lambda, \mu$, the mass density $\rho$, the magnetic permeability $\mu_{0}$, and a parameter $\beta$ which is proportional to the electric conductivity are given by $[6,12,16,18,21]$

$$
\begin{gathered}
\mu \Delta \mathbf{u}(\mathbf{x}, t)+(\lambda+\mu) \nabla \nabla \cdot \mathbf{u}(\mathbf{x}, t)+\frac{\mu_{0}}{4 \pi}[\nabla \times \mathbf{h}(\mathbf{x}, t)] \times \mathbf{H}=\rho \frac{\partial^{2} \mathbf{u}(\mathbf{x}, t)}{\partial t^{2}} \\
\Delta \mathbf{h}(\mathbf{x}, t)-\beta \frac{\partial \mathbf{h}(\mathbf{x}, t)}{\partial t}=-\beta \nabla \times\left[\frac{\partial \mathbf{u}(\mathbf{x}, t)}{\partial t} \times \mathbf{H}\right]
\end{gathered}
$$

where $\mathbf{u}$ denotes the displacement field, $\mathbf{h}$ stands for the generated magnetic field, and $\mathbf{H}$ is the imposed constant magnetic field taken along the $x_{3}$-axis. Following Nowacki [21], besides linearization of the fundamental equations we have also eliminated the displacement current which is proportional to the time derivative of the elastic field since its influence of electromagnetic quantities is very small. We consider the Cauchy problem, where $\mathbf{x}$ varies in $\mathbb{R}^{3}$ and $t>0$ with the Cauchy data

$$
\begin{aligned}
\mathbf{u}(\mathbf{x}, 0) & =\mathbf{u}_{0}(\mathbf{x}), \\
\frac{\partial \mathbf{u}(\mathbf{x}, t)}{\partial t} \mid & =\mathbf{u}_{1}(\mathbf{x}), \\
\mathbf{h}(\mathbf{x}, 0) & =\mathbf{h}_{0}(\mathbf{x}) .
\end{aligned}
$$

The degree of regularity of the data $\mathbf{u}_{0}, \mathbf{u}_{1}, \mathbf{h}_{0}$ will be specified later, where the effects it has on the behaviour of the corresponding energy norms will be demonstrated.

Introducing the spacial Fourier transforms

$$
\begin{aligned}
& \hat{\mathbf{u}}(\boldsymbol{\xi}, t)=(2 \pi)^{-3 / 2} \int_{\mathbb{R}^{3}} e^{i \mathbf{x} \cdot \boldsymbol{\xi}} \mathbf{u}(\mathbf{x}, t) d v(\mathbf{x}), \\
& \hat{\mathbf{h}}(\boldsymbol{\xi}, t)=(2 \pi)^{-3 / 2} \int_{\mathbb{R}^{3}} e^{i \mathbf{x} \cdot \boldsymbol{\xi}} \mathbf{h}(\mathbf{x}, t) d v(\mathbf{x})
\end{aligned}
$$

into the Cauchy problem (1)-(5) we arrive at the following initial-value problem for the spectral magnetoelastic equations:

$$
\begin{aligned}
& \mu r^{2} \hat{\mathbf{u}}(\boldsymbol{\xi}, t)+(\lambda+\mu) r^{2} \mathbf{a} \otimes \mathbf{a} \cdot \hat{\mathbf{u}}(\boldsymbol{\xi}, t) \\
&+i \frac{\mu_{0} r}{4 \pi}[\mathbf{a} \times \hat{\mathbf{h}}(\boldsymbol{\xi}, t)] \times \mathbf{H}+\rho \frac{d^{2} \hat{\mathbf{u}}(\boldsymbol{\xi}, t)}{d t^{2}}=\mathbf{0} \\
& r^{2} \hat{\mathbf{h}}(\boldsymbol{\xi}, t)+\beta \frac{d \hat{\mathbf{h}}(\boldsymbol{\xi}, t)}{d t}+i r \beta \mathbf{a} \times\left[\frac{d \hat{\mathbf{u}}(\boldsymbol{\xi}, t)}{d t} \times \mathbf{H}\right]=\mathbf{0}, \\
& \hat{\mathbf{u}}(\boldsymbol{\xi}, 0)=\hat{\mathbf{u}}_{0}(\boldsymbol{\xi}) \\
&\left.\frac{d \hat{\mathbf{u}}(\boldsymbol{\xi}, t)}{d t}\right|_{t=0}=\hat{\mathbf{u}}_{1}(\boldsymbol{\xi}), \\
& \hat{\mathbf{h}}(\boldsymbol{\xi}, 0)=\hat{\mathbf{h}}_{0}(\boldsymbol{\xi}),
\end{aligned}
$$


where $\hat{\mathbf{u}}_{0}, \hat{\mathbf{u}}_{1}, \hat{\mathbf{h}}_{0}$ denote the Fourier transforms of the Cauchy data and

$$
\boldsymbol{\xi}=r \mathbf{a}, \quad r=|\boldsymbol{\xi}|, \quad \mathbf{a}=\left(a_{1}, a_{2}, a_{3}\right) .
$$

For each $\boldsymbol{\xi} \in \mathbb{R}^{3}$, the initial-value problem (8)-(12) describes the propagation of a coupled magnetoelastic plane wave propagating in the direction $\mathbf{a}$.

If we introduce the phase velocities for the longitudinal and transverse waves

$$
\nu_{p}=\sqrt{\frac{\lambda+2 \mu}{\rho}}, \quad \nu_{s}=\sqrt{\frac{\mu}{\rho}}
$$

respectively, the Alfvén velocity

$$
\nu_{a}=H \sqrt{\frac{\mu_{0}}{4 \pi \rho}},
$$

the exterior magnetic field $\mathbf{H}=H \hat{\mathbf{x}}_{3}$, and eliminate the magnetic field $\hat{\mathbf{h}}$ among (8)-(12) we arrive at

$$
\begin{gathered}
{\left[\mathbb{I} \frac{d^{3}}{d t^{3}}+\frac{r^{2}}{\beta} \mathbb{I} \frac{d^{2}}{d t^{2}}+r^{2}(\mathbb{E}+\mathbb{M}) \frac{d}{d t}+\frac{r^{4}}{\beta} \mathbb{E}\right] \cdot \hat{\mathbf{u}}(\boldsymbol{\xi}, t)=\mathbf{0},} \\
\hat{\mathbf{u}}(\boldsymbol{\xi}, 0)=\hat{\mathbf{u}}_{0}(\boldsymbol{\xi}), \\
\left.\frac{d \hat{\mathbf{u}}(\boldsymbol{\xi}, t)}{d t}\right|_{t=0}=\hat{\mathbf{u}}_{1}(\boldsymbol{\xi}), \\
\left.\frac{d^{2} \hat{\mathbf{u}}(\boldsymbol{\xi}, t)}{d t^{2}}\right|_{t=0}=-r^{2} \mathbb{E} \cdot \hat{\mathbf{u}}_{0}(\boldsymbol{\xi})-i \frac{r}{a_{3} H} \mathbb{M} \cdot \hat{\mathbf{h}}_{0},
\end{gathered}
$$

where $\mathbb{I}$ is the identity dyadic,

$$
\mathbb{E}=\nu_{p}^{2} \mathbf{a} \otimes \mathbf{a}+\nu_{s}^{2}(\mathbb{I}-\mathbf{a} \otimes \mathbf{a})
$$

is the elastic dyadic [8], and

$$
\mathbb{M}=\nu_{a}^{2}\left[\mathbf{a} \otimes \mathbf{a}+a_{3}^{2} \mathbb{I}-a_{3}\left(\mathbf{a} \otimes \hat{\mathbf{x}}_{3}+\hat{\mathbf{x}}_{3} \otimes \mathbf{a}\right)\right]
$$

is the magnetic dyadic [9].

The elastic dyadic $\mathbb{E}$ involves only elastic parameters and decomposes any vector into a component along a and a component perpendicular to $\mathbf{a}$. On the other hand, the magnetic dyadic $\mathbb{M}$ involves only electromagnetic parameters and acts as a projection operator on the $\left(x_{1}, x_{2}\right)$-plane. In fact, $\mathbb{M}$ has the nonionic form

$$
\mathbb{M}=\nu_{a}^{2}\left[\begin{array}{ccc}
a_{1}^{2}+a_{3}^{2} & a_{1} a_{2} & 0 \\
a_{1} a_{2} & a_{2}^{2}+a_{3}^{2} & 0 \\
0 & 0 & 0
\end{array}\right]
$$

In (19) we have used the fact that since $\mathbf{h}(\mathbf{x}, t)$ is the solenoidal field, $\hat{\mathbf{h}}(\boldsymbol{\xi}, t)$ is transverse to $\mathbf{a}$. 
As $\beta \rightarrow+\infty$, Eq. (16) recovers the corresponding equation for the perfectly conducting case [9], while as $\beta \rightarrow 0+$ Eq. (16) reduces to classical elasticity [8]. Any solution of (16)-(19) describes a plane displacement wave propagating along a while the general disturbance is obtained by inverting (6). The coupled magnetic wave is derived by solving either (8) or (9) and, by inversion of (7), the general magnetic disturbance is obtained. Finally, the corresponding electric field is evaluated from the magnetic field with the use of Maxwell's equations.

The vector equation (16) can be written as a $9 \times 9$ first-order system and the characteristic equation of this system is given by the determinant

$$
\left|\begin{array}{ccc}
\tau \mathbb{I} & -\mathbb{I} & \mathbb{O} \\
\mathbb{O} & \tau \mathbb{I} & -\mathbb{I} \\
\frac{r^{4}}{\beta} \mathbb{E} & r^{2}(\mathbb{E}+\mathbb{M}) & \left(\tau+\frac{r^{2}}{\beta}\right) \mathbb{I}
\end{array}\right|=0
$$

where every entry is a $3 \times 3$ matrix representing the nonionic form [5] of the $\mathbb{I}, \mathbb{O}, \mathbb{E}$, and M. Equation (22) has the following expansion as a 9th-degree polynomial:

$$
\begin{aligned}
P_{9}(\tau)=\tau^{9} & +3 \frac{r^{2}}{\beta} \tau^{8}+\left[A_{7}+3\left(\frac{r^{2}}{\beta}\right)^{2}\right] \tau^{7} \\
& +\frac{r^{2}}{\beta}\left[A_{6}+\left(\frac{r^{2}}{\beta}\right)^{2}\right] \tau^{6}+\left[A_{5}+\left(\frac{r^{2}}{\beta}\right)^{2} B_{5}\right] \tau^{5} \\
& +\frac{r^{2}}{\beta}\left[A_{4}+\left(\frac{r^{2}}{\beta}\right)^{2} B_{4}\right] \tau^{4}+\left[A_{3}+\left(\frac{r^{2}}{\beta}\right)^{2} B_{3}\right] \tau^{3} \\
& +\frac{r^{2}}{\beta}\left[A_{2}+\left(\frac{r^{2}}{\beta}\right)^{2} B_{2}\right] \tau^{2}+\left(\frac{r^{2}}{\beta}\right)^{2} B_{1} \tau+\left(\frac{r^{2}}{\beta}\right)^{3} B_{0}=0
\end{aligned}
$$

where

$$
\begin{aligned}
& A_{7}=r^{2}\left[\nu_{p}^{2}+2 \nu_{s}^{2}+\nu_{a}^{2}+\nu_{a}^{2} a_{3}^{2}\right] \\
& A_{6}=r^{2}\left[3 \nu_{p}^{2}+6 \nu_{s}^{2}+2 \nu_{a}^{2}+2 \nu_{a}^{2} a_{3}^{2}\right] \\
& A_{5}=r^{4}\left[2 \nu_{p}^{2} \nu_{s}^{2}+\nu_{s}^{4}+2 \nu_{a}^{2} \nu_{s}^{2}+2 \nu_{p}^{2} \nu_{a}^{2} a_{3}^{2}+\nu_{a}^{4} a_{3}^{2}\right] \\
& B_{5}=r^{2}\left[3 \nu_{p}^{2}+6 \nu_{s}^{2}+\nu_{a}^{2}+\nu_{a}^{2} a_{3}^{2}\right] \\
& A_{4}=r^{4}\left[6 \nu_{p}^{2} \nu_{s}^{2}+3 \nu_{s}^{4}+4 \nu_{s}^{2} \nu_{a}^{2}+4 \nu_{p}^{2} \nu_{a}^{2} a_{3}^{2}+\nu_{a}^{4} a_{3}^{2}\right] \\
& B_{4}=r^{2}\left[\nu_{p}^{2}+2 \nu_{s}^{2}\right] \\
& A_{3}=r^{6}\left[\nu_{p}^{2} \nu_{s}^{4}+\nu_{s}^{4} \nu_{a}^{2}+2 \nu_{p}^{2} \nu_{s}^{2} \nu_{a}^{2} a_{3}^{2}+\nu_{p}^{2} \nu_{a}^{4} a_{3}^{4}-\nu_{s}^{4} \nu_{a}^{2} a_{3}^{2}-\nu_{s}^{2} \nu_{a}^{4} a_{3}^{4}+\nu_{s}^{2} \nu_{a}^{4} a_{3}^{2}\right] \\
& B_{3}=r^{4}\left[6 \nu_{p}^{2} \nu_{s}^{2}+3 \nu_{s}^{4}+2 \nu_{s}^{2} \nu_{a}^{2}+2 \nu_{p}^{2} \nu_{a}^{2} a_{3}^{2}\right] \\
& A_{2}=r^{6}\left[3 \nu_{p}^{2} \nu_{s}^{4}+2 \nu_{s}^{4} \nu_{a}^{2}+4 \nu_{p}^{2} \nu_{s}^{2} \nu_{a}^{2} a_{3}^{2}+\nu_{p}^{2} \nu_{a}^{4} a_{3}^{4}-2 \nu_{s}^{4} \nu_{a}^{2} a_{3}^{2}-\nu_{s}^{2} \nu_{a}^{4} a_{3}^{4}+\nu_{s}^{2} \nu_{a}^{4} a_{3}^{2}\right] \\
& B_{2}=r^{4}\left[2 \nu_{p}^{2} \nu_{s}^{2}+\nu_{s}^{4}\right] \\
& B_{1}=r^{6}\left[2 \nu_{s}^{4} \nu_{a}^{2}+2 \nu_{p}^{2} \nu_{s}^{2} \nu_{a}^{2} a_{3}^{2}-\nu_{s}^{4} \nu_{a}^{2} a_{3}^{2}\right] \\
& B_{0}=r^{6} \nu_{p}^{2} \nu_{s}^{4}
\end{aligned}
$$


The 9 roots of (23) are needed in order to form the solution of Eq. (16). We will show in the following section that an appropriate physical interpretation of the coupling will lead itself to a factorization of (23) into three polynomials of degree one, three, and five. For a perfect conductor, Eq. (23) is reduced to

$$
\tau^{3}\left(\tau^{6}+A_{7} \tau^{4}+A_{5} \tau^{2}+A_{3}\right)=0
$$

which is readily solvable [9].

3. Geometrical decomposition and physical coupling. In the classical theory of dynamic elasticity, for a homogeneous and isotropic medium, the irrotational (longitudinal) waves propagate independently of the solenoidal (transverse) waves as long as the waves do not meet a boundary, in which case a mode conversion takes place $[12,16,21]$. As we explain in the sequel, this is not true when the waves propagate in the presence of an externally imposed magnetic field. In order to explain the coupling, on the basis of physical arguments, we consider the external constant magnetic field along the $x_{3}$-axis and an elastic plane wave propagating in the direction a. If the elastic plane wave is of the longitudinal type then the motion occurs along the direction of propagation $\mathbf{a}$. The Lorentz force is directed along $\mathbf{a} \times \mathbf{H}$, i.e., along a vector perpendicular to the meridian plane through a, which generates a transverse wave. Therefore, the existence of the magnetic field $\mathbf{H}$ forces the longitudinal wave to couple with a component of the transverse wave. Only plane longitudinal waves that propagate along the direction of $\mathbf{H}$ will avoid coupling with the transverse wave, since then no Lorentz force is generated. On the other hand, a plane transverse wave, propagating along a and polarized along $\mathbf{P}$ gives rise to a Lorentz force in the direction $\mathbf{P} \times \mathbf{H}$, which, in general, has a component along a. This longitudinal component is responsible for the coupling of the transverse with the longitudinal wave. The only waves that do not suffer of this coupling are transverse waves that are propagating in directions perpendicular to $\mathbf{H}$ and are polarized along $\mathbf{H}$.

Therefore, the magnetoelastic coupling is a generic property of the set of plane waves which is established as follows. Any elastic disturbance is decomposed into a longitudinal and a transverse part. Each one of these parts is decomposed into plane waves. A longitudinal plane wave generates, through the Lorentz force, a transverse component and vice versa, a transverse plane wave generates, for the same reason, a longitudinal component. These two kings of components establish the coupling forced by the external magnetic field.

In order to further analyze this coupling we introduce a spherical coordinate system based on the direction of propagation

$$
\mathbf{a}=\hat{\mathbf{r}}=(\sin \theta \cos \varphi, \sin \theta \sin \varphi, \cos \theta)
$$

Then, for a longitudinal wave, as a sweeps the meridian plane $\varphi=$ constant, the Lorentz force $\mathbf{a} \times \mathbf{H}$ preserves its direction while its magnitude varies proportional to the nonlinear function $\sin \theta$. On the other hand, as a rotates around $\mathbf{H}$, generating the cone $\theta=$ constant, the magnitude of the Lorentz force remains invariant and its direction rotates around $\mathbf{H}$ on the $\left(x_{1}, x_{2}\right)$-plane. 
For a transverse wave, a similar nonlinear coupling holds. Decomposing the system (8), (9) into the spherical directions $\hat{\mathbf{r}}=\mathbf{a}, \hat{\boldsymbol{\theta}}, \hat{\boldsymbol{\varphi}}$ with

$$
\begin{aligned}
& \hat{\mathbf{u}}=\hat{u}_{r} \hat{\mathbf{r}}+\hat{u}_{\theta} \hat{\boldsymbol{\theta}}+\hat{u}_{\varphi} \hat{\boldsymbol{\varphi}}, \\
& \hat{\mathbf{h}}=\hat{h}_{r} \hat{\mathbf{r}}+\hat{h}_{\theta} \hat{\boldsymbol{\theta}}+\hat{h}_{\varphi} \hat{\boldsymbol{\varphi}}
\end{aligned}
$$

we arrive at the following three partially decoupled systems: the radial equation

$$
\frac{d \hat{h}_{r}}{d t}+\frac{r^{2}}{\beta} \hat{h}_{r}=0
$$

for the radial component of $\hat{\mathbf{h}}$; the azimuthal system

$$
\begin{aligned}
\frac{d^{2} \hat{u}_{\varphi}}{d t^{2}}+r^{2} \nu_{s}^{2} \hat{u}_{\varphi} & =-i r \frac{\nu_{a}^{2} a_{3}}{H} \hat{h}_{\varphi} \\
\frac{d \hat{h}_{\varphi}}{d t}+\frac{r^{2}}{\beta} \hat{h}_{\varphi} & =-i r H a_{3} \frac{d \hat{u}_{\varphi}}{d t}
\end{aligned}
$$

that couples the azimuthal components of $\hat{\mathbf{u}}$ and $\hat{\mathbf{h}}$; and the meridian system

$$
\begin{aligned}
\frac{d^{2} \hat{u}_{r}}{d t^{2}}+r^{2} \nu_{p}^{2} \hat{u}_{r} & =-i r \frac{\nu_{a}^{2}}{H} \sqrt{1-a_{3}^{2}} \hat{h}_{\theta}, \\
\frac{d^{2} \hat{u}_{\theta}}{d t^{2}}+r^{2} \nu_{s}^{2} \hat{u}_{\theta} & =-i r \frac{\nu_{a}^{2} a_{3}}{H} \hat{h}_{\theta}, \\
\frac{d \hat{h}_{\theta}}{d t}+\frac{r^{2}}{\beta} \hat{h}_{\theta} & =-i r H\left[\sqrt{1-a_{3}^{2}} \frac{d \hat{u}_{r}}{d t}+a_{3} \frac{d \hat{u}_{\theta}}{d t}\right],
\end{aligned}
$$

which connects the meridian components of $\hat{\mathbf{u}}$ with the $\theta$ component of $\hat{\mathbf{h}}$.

Equation (40) describes the attenuation of the radial component of $\hat{\mathbf{h}}$. As the conductivity increases, the attenuation decreases, and in the limit as $\beta \rightarrow+\infty$ (perfect conductor), the radial components of $\hat{\mathbf{h}}$ remain constant.

The coupling established by the system (41), (42) concerns the transverse wave and the magnetic field, while the highest-order coupling is present in the system (43), (44), (45), where both longitudinal and transverse waves are coupled with the magnetic field.

Eliminating $\hat{h}_{\varphi}$ between (41) and (42) we obtain

$$
\frac{d^{3} \hat{u}_{\varphi}}{d t^{3}}+\frac{r^{2}}{\beta} \frac{d^{2} \hat{u}_{\varphi}}{d t^{2}}+r^{2}\left(\nu_{s}^{2}+\nu_{a}^{2} a_{3}^{2}\right) \frac{d \hat{u}_{\varphi}}{d t}+\frac{r^{4} \nu_{s}^{2}}{\beta} \hat{u}_{\varphi}=0
$$

for the azimuthal component of $\hat{\mathbf{u}}$. Similarly, for the system (43)-(45), elimination of $\hat{\mathbf{h}}_{\theta}$ yields the two equations

$$
\left[\frac{d^{2} \hat{u}_{r}}{d t^{2}}+r^{2} \nu_{p}^{2} \hat{u}_{r}\right] a_{3}=\left[\frac{d^{2} \hat{u}_{\theta}}{d t^{2}}+r^{2} \nu_{s}^{2} \hat{u}_{\theta}\right] \sqrt{1-a_{3}^{2}}
$$

and

$$
\frac{d^{3} \hat{u}_{r}}{d t^{3}}+\frac{r^{2}}{\beta} \frac{d^{2} \hat{u}_{r}}{d t^{2}}+r^{2}\left[\nu_{p}^{2}+\nu_{a}^{2}\left(1-a_{3}^{2}\right)\right] \frac{d \hat{u}_{r}}{d t}+\frac{r^{4} \nu_{p}^{2}}{\beta} \hat{u}_{r}=-r^{2} \nu_{a}^{2} a_{3} \sqrt{1-a_{3}^{2}} \frac{d \hat{u}_{\theta}}{d t}
$$


or Eq. (47) and the equivalent equation

$$
\frac{d^{3} \hat{u}_{\theta}}{d t^{3}}+\frac{r^{2}}{\beta} \frac{d^{2} \hat{u}_{\theta}}{d t^{2}}+r^{2}\left[\nu_{s}^{2}+\nu_{a}^{2} a_{3}^{2}\right] \frac{d \hat{u}_{\theta}}{d t}+\frac{r^{4} \nu_{s}^{2}}{\beta} \hat{u}_{\theta}=-r^{2} \nu_{a}^{2} a_{3} \sqrt{1-a_{3}^{2}} \frac{d \hat{u}_{r}}{d t},
$$

which can be obtained from (48) if we perform the interchanges

$$
\begin{aligned}
& \hat{u}_{r} \leftrightarrow \hat{u}_{\theta}, \\
& \nu_{p} \leftrightarrow \nu_{s}, \\
& a_{3} \leftrightarrow \sqrt{1-a_{3}^{2}} .
\end{aligned}
$$

A further elimination of $\hat{u}_{\theta}$ between (47) and (48) yields the fifth-order equation

$$
\begin{aligned}
\frac{d^{5} \hat{u}_{r}}{d t^{5}}+\frac{r^{2}}{\beta} \frac{d^{4} \hat{u}_{r}}{d t^{4}}+r^{2}\left[\nu_{p}^{2}\right. & \left.+\nu_{s}^{2}+\nu_{a}^{2}\right] \frac{d^{3} \hat{u}_{r}}{d t^{3}}+\frac{r^{4}}{\beta}\left[\nu_{p}^{2}+\nu_{s}^{2}\right] \frac{d^{2} \hat{u}_{r}}{d t^{2}} \\
& +r^{4}\left[\nu_{p}^{2}\left(\nu_{s}^{2}+\nu_{a}^{2} a_{3}^{2}\right)+\nu_{s}^{2} \nu_{a}^{2}\left(1-a_{3}^{2}\right)\right] \frac{d \hat{u}_{r}}{d t}+\frac{r^{6}}{\beta} \nu_{p}^{2} \nu_{s}^{2} \hat{u}_{r}=0
\end{aligned}
$$

for the radial component of $\hat{\mathbf{u}}$. Both equations (46) and (51) can easily reduce their order by one if the medium exhibits perfect conductivity [9].

If Eqs. (40), (46), and (51) are solved, the fields $\hat{h}_{r}, \hat{u}_{\varphi}$, and $\hat{u}_{r}$ are obtained. Then Eqs. (41), (43) provide $\hat{h}_{\varphi}, \hat{h}_{\theta}$, respectively, and Eq. (45) provides $\hat{u}_{\theta}$. Consequently, Eq. (40), the system (41), (42), and the system (43), (44), (45), or equivalently (40), (46), (51), form an equivalent decomposition of Eq. (16). Equations (40), (46), and (51) have the characteristic polynomials

$$
\begin{aligned}
& P_{1}(\tau)=\tau+\frac{r^{2}}{\beta} \\
& P_{3}(\tau)=\tau^{3}+\frac{r^{2}}{\beta} \tau^{2}+r^{2}\left(\nu_{s}^{2}+\nu_{a}^{2} a_{3}^{2}\right) \tau+\frac{r^{4} \nu_{s}^{2}}{\beta}
\end{aligned}
$$

and

$$
\begin{aligned}
P_{5}(\tau)= & \tau^{5}+\frac{r^{2}}{\beta} \tau^{4}+r^{2}\left(\nu_{p}^{2}+\nu_{s}^{2}+\nu_{a}^{2}\right) \tau^{3}+\frac{r^{4}\left(\nu_{p}^{2}+\nu_{s}^{2}\right)}{\beta} \tau^{2} \\
& +r^{4}\left[\nu_{p}^{2} \nu_{s}^{2}+\nu_{s}^{2} \nu_{a}^{2}+\nu_{p}^{2} \nu_{a}^{2} a_{3}^{2}-\nu_{s}^{2} \nu_{a}^{2} a_{3}^{2}\right] \tau+\frac{r^{6} \nu_{p}^{2} \nu_{s}^{2}}{\beta}
\end{aligned}
$$

It is easily checked that the characteristic polynomial (23) of Eq. (16) accepts the factorization

$$
P_{9}(\tau)=P_{1}(\tau) P_{3}(\tau) P_{5}(\tau)
$$

which establishes the spherical decomposition discussed above.

The fact that the Lorentz force does not allow any further decoupling of the systems (41), (42) and (43), (44), (45) is reflected upon the irreducibility of the polynomials $P_{3}$ and $P_{5}$ respectively, within the real number field. 
4. Asymptotic behaviour of the characteristic roots. The characteristic polynomial $P_{9}$ represents the dispersion relation for the propagation of magnetoelastic waves, connecting the wave number $r$ (dual spacial variable) with the frequency $\tau$ (dual temporal variable). In order to determine the roots of the characteristic equation we need to solve the three polynomial equations

$$
P_{i}(\tau)=0, \quad i=1,3,5
$$

For $i$ equal to 1 and 3 the roots are easily obtained, but since $P_{5}$ is irreducible, Eq. (56), for $i$ equal to 5 , cannot be solved in a finite number of steps. Note that, as Hermite [13] has shown, the five roots of the reduced quintic form, introduced by Jerrard [14], can be expressed in terms of elliptic integrals. Nevertheless, we do not need to go through this extremely complicated algorithm since, for our purpose, we only need to derive the asymptotic behaviour of the 9 roots for $r$ in a neighborhood of the origin (low-frequency regime) and in a neighborhood of infinity (high-frequency regime).

We introduce the dimensionless nonnegative parameters

$$
\begin{aligned}
& c_{p}=\frac{\nu_{a}^{2}}{\nu_{p}^{2}}\left(1-a_{3}^{2}\right), \\
& c_{s}=\frac{\nu_{a}^{2}}{\nu_{s}^{2}} a_{3}^{2}
\end{aligned}
$$

and the scaling

$$
\tau=r \lambda=\bar{r} \beta \lambda
$$

Then the cubic and the quintic equations are written as

$$
\lambda^{3}+\bar{r} \lambda^{2}+\nu_{s}^{2}\left(1+c_{s}\right) \lambda+\bar{r} v_{s}^{2}=0
$$

and

$$
\begin{gathered}
\lambda^{5}+\bar{r} \lambda^{4}+\left(\nu_{p}^{2}+\nu_{s}^{2}+\nu_{a}^{2}\right) \lambda^{3}+\bar{r}\left(\nu_{p}^{2}+\nu_{s}^{2}\right) \lambda^{2} \\
+\nu_{p}^{2} \nu_{s}^{2}\left(1+c_{p}+c_{s}\right) \lambda+\bar{r} \nu_{p}^{2} \nu_{s}^{2}=0
\end{gathered}
$$

where all dimensional variables have the same dimensions.

In order to obtain the asymptotic forms of the roots at the origin we use regular perturbation theory [3, chapter 7] considering the asymptotic series

$$
\lambda(\bar{r})=\sum_{n=0}^{\infty} \beta_{n} \bar{r}^{n}
$$

and evaluating a few first coefficients. After some long and tedious calculations we obtain the following forms, as $\bar{r} \rightarrow 0+$,

$$
\begin{aligned}
& \lambda_{2}(\bar{r})=-\frac{1}{1+c_{s}} \bar{r}+O\left(\bar{r}^{2}\right), \\
& \lambda_{3}(\bar{r})=\lambda_{4}^{*}(\bar{r})+i \nu_{s} \sqrt{1+c_{s}}-\frac{c_{s}}{2\left(1+c_{s}\right)} \bar{r}+O\left(\bar{r}^{2}\right)
\end{aligned}
$$


for the roots of the cubic, and

$$
\begin{aligned}
& \lambda_{5}(\bar{r})=-\frac{1}{1+c_{p}+c_{s}} \bar{r}+O\left(\bar{r}^{2}\right), \\
& \lambda_{6}(\bar{r})=\lambda_{7}^{*}(\bar{r})=i \gamma_{+}+\frac{\nu_{p}^{2} \nu_{s}^{2}\left(c_{p}+c_{s}\right)-\nu_{a}^{2} \gamma_{+}^{2}}{2\left(\nu_{p}^{2}+\nu_{s}^{2}+\nu_{a}^{2}\right) \gamma_{+}^{2}-4 \nu_{p}^{2} \nu_{s}^{2}\left(1+c_{p}+c_{s}\right)} \bar{r}+O\left(\bar{r}^{2}\right), \\
& \lambda_{8}(\bar{r})=\lambda_{9}^{*}(\bar{r})+i \gamma_{-}+\frac{\nu_{p}^{2} \nu_{s}^{2}\left(c_{p}+c_{s}\right)-\nu_{a}^{2} \gamma_{-}^{2}}{2\left(\nu_{p}^{2}+\nu_{s}^{2}+\nu_{a}^{2}\right) \gamma_{-}^{2}-4 \nu_{p}^{2} \nu_{s}^{2}\left(1+c_{p}+c_{s}\right)} \bar{r}+O\left(\bar{r}^{2}\right)
\end{aligned}
$$

for the roots of the quintic equation, where

$$
2 \gamma_{ \pm}^{2}=\left(\nu_{p}^{2}+\nu_{s}^{2}+\nu_{a}^{2}\right) \pm\left[\left(\nu_{p}^{2}-\nu_{s}^{2}+\nu_{a}^{2}\right)^{2}-4\left(\nu_{p}^{2}-\nu_{s}^{2}\right) \nu_{a}^{2} a_{3}^{2}\right]^{1 / 2} .
$$

The expressions $\gamma_{+}^{2}$ and $\gamma_{-}^{2}$ coincide with the roots $\lambda_{2}^{2}$ and $\lambda_{3}^{2}$ in the case of perfect conductivity [9].

Therefore, in a neighborhood of the origin, the nine roots of $P_{9}(\tau)$ behave as follows:

$$
\begin{aligned}
& \tau_{1}(r)=-\frac{r^{2}}{\beta}, \\
& \tau_{2}(r)=-\frac{1}{1+c_{s}} \frac{r^{2}}{\beta}+O\left(r^{4}\right), \\
& \tau_{3}(r)=\tau_{4}^{*}(r)=i \nu_{s} \sqrt{1+c_{s}} r-\frac{c_{s}}{2\left(1+c_{s}\right)} \frac{r^{2}}{\beta}+O\left(r^{3}\right), \\
& \tau_{5}(r)=-\frac{1}{1+c_{p}+c_{s}} \frac{r^{2}}{\beta}+O\left(r^{4}\right), \\
& \tau_{6}(r)=\tau_{7}^{*}(r)=i \gamma_{+} r+\frac{\nu_{p}^{2} \nu_{s}^{2}\left(c_{p}+c_{s}\right)-\nu_{a}^{2} \gamma_{+}^{2}}{2\left(\nu_{p}^{2}+\nu_{s}^{2}+\nu_{a}^{2}\right) \gamma_{+}^{2}-4 \nu_{p}^{2} \nu_{s}^{2}\left(1+c_{p}+c_{s}\right)} \frac{r^{2}}{\beta}+O\left(r^{3}\right), \\
& \tau_{8}(r)=\tau_{9}^{*}(r)=i \gamma_{-} r+\frac{\nu_{p}^{2} \nu_{s}^{2}\left(c_{p}+c_{s}\right)-\nu_{a}^{2} \gamma_{-}^{2}}{2\left(\nu_{p}^{2}+\nu_{s}^{2}+\nu_{a}^{2}\right) \gamma_{-}^{2}-4 \nu_{p}^{2} \nu_{s}^{2}\left(1+c_{p}+c_{s}\right)} \frac{r^{2}}{\beta}+O\left(r^{3}\right) .
\end{aligned}
$$

For the asymptotic behavior as $r \rightarrow+\infty$ we use singular perturbation theory and dominant balance arguments $[3$, chapter 7$]$ to derive the forms

$$
\begin{aligned}
& \lambda_{2}(\bar{r})=-\bar{r}+O(1), \\
& \lambda_{3}(\bar{r})=\lambda_{4}^{*}(\bar{r})=i \nu_{s}+O\left(\frac{1}{\bar{r}}\right)
\end{aligned}
$$

for the roots of the cubic equation (59), and

$$
\begin{aligned}
& \lambda_{5}(\bar{r})=-\bar{r}+O(1), \\
& \lambda_{6}(\bar{r})=\lambda_{7}^{*}(\bar{r})=i \nu_{s}+O\left(\frac{1}{\bar{r}}\right), \\
& \lambda_{8}(\bar{r})=\lambda_{9}^{*}(\bar{r})=i \nu_{p}+O\left(\frac{1}{\bar{r}}\right)
\end{aligned}
$$

for the roots of the quintic equation (60). 
Consequently, in a neighborhood of infinity the following asymptotic behavior is established:

$$
\begin{aligned}
& \tau_{1}(r)=-\frac{r^{2}}{\beta}, \\
& \tau_{2}(r)=-\frac{r^{2}}{\beta}+O(1), \\
& \tau_{3}(r)=\tau_{4}^{*}(r)=i \nu_{s} r+O(1), \\
& \tau_{5}(r)=-\frac{r^{2}}{\beta}+O(1), \\
& \tau_{6}(r)=\tau_{7}^{*}(r)=i \nu_{s} r+O(1), \\
& \tau_{8}(r)=\tau_{9}^{*}(r)=i \nu_{p} r+O(1) .
\end{aligned}
$$

Note that the real roots $\tau_{1}, \tau_{2}, \tau_{5}$ provide a higher, than the other roots, dissipation in a medium that has finite conductivity. The above asymptotic forms will be used later to evaluate the asymptotic behavior of the energy integrals.

5. The canonical form of the solution. In this section we are going to use the standard form [15] of the Cauchy problems and the theory of invariants for dyadics [5] in order to obtain the solution of (16)-(19) in its canonical form. Following John [15, chapter 5 ] the solution $\hat{\mathbf{u}}$ of $(16)-(19)$, with the $\boldsymbol{\xi}$ variable suppressed, is expressed in the form

$$
\hat{\mathbf{u}}(t)=\left[\mathbb{I} \frac{d^{2}}{d t^{2}}+\mathbb{I} \frac{r^{2}}{\beta} \frac{d}{d t}+r^{2}(\mathbb{E}+\mathbb{M})\right] \cdot \hat{\mathbf{u}}_{1}(t)+\left[\frac{d}{d t}+\frac{r^{2}}{\beta}\right] \hat{\mathbf{u}}_{2}(t)+\hat{\mathbf{u}}_{3}(t)
$$

where the vector fields $\hat{\mathbf{u}}_{1}, \hat{\mathbf{u}}_{2}, \hat{\mathbf{u}}_{3}$ solve Eq. (16) and satisfy the following standard Cauchy data:

$$
\begin{aligned}
\hat{\mathbf{u}}_{1}(0) & =\mathbf{0} \\
\left.\frac{d \hat{\mathbf{u}}_{1}(t)}{d t}\right|_{t=0} & =\mathbf{0} \\
\left.\frac{d^{2} \hat{\mathbf{u}}_{1}(t)}{d t^{2}}\right|_{t=0} & =\hat{\mathbf{u}}_{0}(\boldsymbol{\xi}), \\
\hat{\mathbf{u}}_{2}(0) & =\mathbf{0}, \\
\left.\frac{d \hat{\mathbf{u}}_{2}(t)}{d t}\right|_{t=0} & =\mathbf{0}, \\
\left.\frac{d^{2} \hat{\mathbf{u}}_{2}(t)}{d t^{2}}\right|_{t=0} & =\hat{\mathbf{u}}_{1}(\boldsymbol{\xi}),
\end{aligned}
$$

and

$$
\begin{aligned}
\hat{\mathbf{u}}_{3}(0) & =\mathbf{0} \\
\left.\frac{d \hat{\mathbf{u}}_{3}(t)}{d t}\right|_{t=0} & =\mathbf{0} \\
\left.\frac{d^{2} \hat{\mathbf{u}}_{3}(t)}{d t^{2}}\right|_{t=0} & =-r^{2} \mathbb{E} \cdot \hat{\mathbf{u}}_{0}(\boldsymbol{\xi})-i r \frac{\nu_{a}^{2}}{H}\left[a_{3} \mathbb{I}-\mathbf{a} \otimes \hat{\mathbf{x}}_{3}\right] \cdot \hat{\mathbf{h}}_{0}(\boldsymbol{\xi}) .
\end{aligned}
$$


The elastic dyadic $\mathbb{E}$ is given by (20) and the magnetic dyadic $\mathbb{M}$ is given by (21). The above standard problems can be solved with the help of the Laplace transform and, substituting everything back into (85), we arrive at the representation

$$
\hat{\mathbf{u}}(t)=\frac{1}{2 \pi i} \int_{\Gamma} e^{\tau t} \mathbb{P}^{-1}(\tau, r) \cdot \mathbf{G}(\tau, r) d \tau
$$

where

$$
\mathbb{P}(\tau, r)=\mathbb{I} \tau^{3}+\mathbb{I} \frac{r^{2}}{\beta} \tau^{2}+r^{2}(\mathbb{E}+\mathbb{M}) \tau+\frac{r^{2}}{\beta} \mathbb{E}
$$

is the characteristic dyadic of Eq. (16), $\Gamma$ is a closed contour in $\mathbb{C}$, depending on $r$, that encloses all 9 roots of the characteristic polynomial and

$$
\begin{gathered}
P_{9}(\tau)=\operatorname{det} \mathbb{P}(\tau, r) \\
\mathbf{G}(\tau, r)=\left(\tau+\frac{r^{2}}{\beta}\right)\left(\tau \hat{\mathbf{u}}_{0}(\boldsymbol{\xi})+\hat{\mathbf{u}}_{1}(\boldsymbol{\xi})\right)+\mathbb{M} \cdot\left(r^{2} \hat{\mathbf{u}}_{0}(\boldsymbol{\xi})-i \frac{r}{a_{3} H} \hat{\mathbf{h}}_{0}(\boldsymbol{\xi})\right)
\end{gathered}
$$

is a vector quadratic polynomial in $\tau$ that incorporates the Cauchy data. We next evaluate the canonical form of the characteristic dyadic $\mathbb{P}$ using the theory of invariants [5]. In the dextral orthonormal system $\mathbf{a}, \mathbf{b}, \mathbf{c}$ where

$$
\begin{aligned}
\mathbf{a} & =\text { propagation vector } \\
\sqrt{1-a_{3}^{2}} \mathbf{b} & =\mathbf{a} \times \hat{\mathbf{x}}_{3} \\
\sqrt{1-a_{3}^{2}} \mathbf{c} & =\mathbf{a} \times\left(\mathbf{a} \times \hat{\mathbf{x}}_{3}\right)
\end{aligned}
$$

for any wave that does not propagate along the polar directions, we express $\mathbb{P}$ in the form

$$
\mathbb{P}=\mathbf{a} \otimes(A \mathbf{a}+C \mathbf{c})+\mathbf{b} \otimes(B \mathbf{b})+\mathbf{c} \otimes(C \mathbf{a}+B \mathbf{c})
$$

where

$$
\begin{aligned}
& A=A(\tau)=\left(\tau+\frac{r^{2}}{\beta}\right)\left(\tau^{2}+r^{2} \nu_{p}^{2}\right)+\tau r^{2} \nu_{a}^{2}\left(1-a_{3}^{2}\right) \\
& B=B(\tau)=\left(\tau+\frac{r^{2}}{\beta}\right)\left(\tau^{2}+r^{2} \nu_{s}^{2}\right)+\tau r^{2} \nu_{a}^{2} a_{3}^{2} \\
& C=C(\tau)=\tau r^{2} \nu_{a}^{2} a_{3} \sqrt{1-a_{3}^{2}}
\end{aligned}
$$

The third scalar invariant of $\mathbb{P}$ yields the relation

$$
P_{9}(\tau)=B\left(A B-C^{2}\right)=P_{1}(\tau) P_{3}(\tau) P_{5}(\tau)
$$

where $P_{1}, P_{3}, P_{5}$ are given in (52), (53), (54) respectively. Calculations lead to the second dyadic invariant

$$
\mathbb{P}_{2}=B^{2} \mathbf{a} \otimes \mathbf{a}+\left(A B-C^{2}\right) \mathbf{b} \otimes \mathbf{b}+A B \mathbf{c} \otimes \mathbf{c}-B C(\mathbf{a} \otimes \mathbf{c}+\mathbf{c} \otimes \mathbf{a})
$$


and by symmetry we arrive at

$$
\mathbb{P}^{-1}=\frac{B}{A B-C^{2}} \mathbf{a} \otimes \mathbf{a}+\frac{1}{B} \mathbf{b} \otimes \mathbf{b}+\frac{A}{A B-C^{2}} \mathbf{c} \otimes \mathbf{c}-\frac{C}{A B-C^{2}}(\mathbf{a} \otimes \mathbf{c}+\mathbf{c} \otimes \mathbf{a}) .
$$

The dyadic $\mathbb{P}$ (and therefore $\mathbb{P}^{-1}$ ) is symmetric. Therefore, it can be expressed in a diagonal canonical form.

The characteristic values of $\mathbb{P}$ are given by

$$
\begin{aligned}
& \mu_{1}(\tau)=\frac{1}{2}(A+B+D) \\
& \mu_{2}(\tau)=B \\
& \mu_{3}(\tau)=\frac{1}{2}(A+B+D)
\end{aligned}
$$

where

$$
D^{2}=(A-B)^{2}+4 C^{2}
$$

and the corresponding normalized invariant directions are given by

$$
\begin{aligned}
& \gamma_{1}(\tau)=\frac{2 C \mathbf{a}-(A-B-D) \mathbf{c}}{\|2 C \mathbf{a}-(A-B-D) \mathbf{c}\|}, \\
& \gamma_{2}(\tau)=\mathbf{b} \\
& \gamma_{3}(\tau)=\frac{2 C \mathbf{a}-(A-B+D) \mathbf{c}}{\|2 C \mathbf{a}-(A-B+D) \mathbf{c}\|} .
\end{aligned}
$$

We see that the invariant direction $\gamma_{2}$ coincides with the direction of the Lorentz force for longitudinal waves and the other two directions are obtained through a rotation around this direction $\gamma_{2}$. The direction $\gamma_{2}(\tau)$ is perpendicular to $\gamma_{1}\left(\tau^{\prime}\right)$ and to $\gamma_{3}\left(\tau^{\prime \prime}\right)$ for any $\tau^{\prime}$ and $\tau^{\prime \prime}$, while $\gamma_{1}\left(\tau^{\prime}\right)$ and $\gamma_{3}\left(\tau^{\prime \prime}\right)$ are perpendicular only when $\tau^{\prime}=\tau^{\prime \prime}$. Consequently, $\gamma_{2}$ is "strongly" orthogonal to $\gamma_{1}$ and $\gamma_{3}$. By virtue of (98), (102), and

$$
\mu_{1} \mu_{2} \mu_{3}=P_{1} P_{3} P_{5}
$$

the canonical forms

$$
\mathbb{P}=\frac{P_{1} P_{5}}{\mu_{3}} \gamma_{1} \otimes \gamma_{1}+P_{3} \gamma_{2} \otimes \gamma_{2}+\frac{P_{1} P_{5}}{\mu_{1}} \gamma_{3} \otimes \gamma_{3}
$$

and

$$
\mathbb{P}^{-1}=\frac{\mu_{3}}{P_{1} P_{5}} \gamma_{1} \otimes \gamma_{1}+\frac{1}{P_{3}} \gamma_{2} \otimes \gamma_{2}+\frac{\mu_{1}}{P_{1} P_{5}} \gamma_{3} \otimes \gamma_{3}
$$

are deduced. The diagonal forms (105), (106) establish the coupling between the longitudinal and the transverse waves imposed by the exterior magnetic field as it is discussed in Sec. 3 .

If the form (106) is introduced in the general expression (89) for the solution and if Cauchy's residue theory is used to evaluate the integrals, we arrive at the following expression for the solution of (16)-(19):

$$
\begin{aligned}
\hat{\mathbf{u}}(r \mathbf{a}, t)=\sum_{j=1,5,6,7,8,9} \Lambda_{1}\left(\tau_{j}\right) e^{\tau_{j} t} \mathbf{G}\left(\tau_{j}, r\right) \cdot \gamma_{1} \otimes \gamma_{1} \\
+\sum_{j=2,3,4} \Lambda_{2}\left(\tau_{j}\right) e^{\tau_{j} t} \mathbf{G}\left(\tau_{j}, r\right) \cdot \gamma_{2} \otimes \gamma_{2} \\
+\sum_{j=1,5,6,7,8,9} \Lambda_{3}\left(\tau_{j}\right) e^{\tau_{j} t} \mathbf{G}\left(\tau_{j}, r\right) \cdot \gamma_{3} \otimes \gamma_{3}
\end{aligned}
$$


where

$$
\begin{array}{lll}
\Lambda_{1}\left(\tau_{j}\right) & =\lim _{\tau \rightarrow \tau_{j}} \frac{\tau-\tau_{j}}{P_{1}(\tau) P_{5}(\tau)} \mu_{3}(\tau), & j=1,5,6,7,8,9, \\
\Lambda_{2}\left(\tau_{j}\right)=\lim _{\tau \rightarrow \tau_{j}} \frac{\tau-\tau_{j}}{P_{3}(\tau)}, & j=2,3,4, \\
\Lambda_{3}\left(\tau_{j}\right)=\lim _{\tau \rightarrow \tau_{j}} \frac{\tau-\tau_{j}}{P_{1}(\tau) P_{5}(\tau)} \mu_{1}(\tau), & j=1,5,6,7,8,9 .
\end{array}
$$

We can easily see that the root $\mu_{1}$ as well as the expression $\gamma_{1} \cdot \mathbb{M}$ vanish when we replace $\tau$ by $-\frac{r^{2}}{\beta}$. Consequently, the first and the last sum in the right-hand side of (107) have a removable singularity at the root of $P_{1}(\tau)$. In view of this observation, the $j=1$ terms in (107) can be omitted and we obtain

$$
\begin{gathered}
\hat{\mathbf{u}}(r \mathbf{a}, t)=\sum_{j=5,6,7,8,9}\left(\Lambda_{1}\left(\tau_{j}\right) \boldsymbol{\gamma}_{1} \otimes \gamma_{1}+\Lambda_{3}\left(\tau_{j}\right) \boldsymbol{\gamma}_{3} \otimes \boldsymbol{\gamma}_{3}\right) \cdot \mathbf{G}\left(\tau_{j}, r\right) e^{\tau_{j} t} \\
\sum_{j=2.3 .4} \Lambda_{2}\left(\tau_{j}\right) \boldsymbol{\gamma}_{2} \otimes \boldsymbol{\gamma}_{2} \cdot \mathbf{G}\left(\tau_{j}, r\right) e^{\tau_{j} t}
\end{gathered}
$$

From (8) and Maxwell's equation $\nabla \cdot \mathbf{h}=0$, which implies the orthogonality relation $\mathbf{a} \cdot \hat{\mathbf{h}}=0$, we reduce, after some dyadic calculations, the expression

$$
\hat{\mathbf{h}}(r \mathbf{a}, t)=\frac{i H}{r \nu_{a}^{2} a_{3}}\left(\frac{d^{2}}{d t^{2}}+\nu_{s}^{2} r^{2}\right)[(\mathbb{I}-\mathbf{a} \otimes \mathbf{a}) \cdot \hat{\mathbf{u}}(r \mathbf{a}, t)]
$$

which furnishes $\hat{\mathbf{h}}$ in terms of the transverse component of $\hat{\mathbf{u}}$.

6. The energies. Multiplying Eq. (1) by the time derivative of $\mathbf{u}$ and using appropriate dyadic identities as well as Eq. (2) we arrive at the local energy law

$$
\begin{aligned}
\frac{\partial}{\partial t}[ & \left.\frac{\rho}{2}\left|\frac{\partial \mathbf{u}}{\partial t}\right|^{2}+\frac{\mu}{2} \nabla \mathbf{u}: \nabla \mathbf{u}+\frac{\lambda+\mu}{2}|\nabla \cdot \mathbf{u}|^{2}+\frac{\mu_{0}}{8 \pi}|\mathbf{h}|^{2}\right] \\
= & \nabla \cdot\left[\mu(\nabla \mathbf{u}) \cdot \frac{\partial \mathbf{u}}{\partial t}+(\lambda+\mu)(\nabla \cdot \mathbf{u}) \frac{\partial \mathbf{u}}{\partial t}+\frac{\mu_{0}}{4 \pi} \mathbf{h} \times\left(\mathbf{H} \times \frac{\partial \mathbf{u}}{\partial t}\right)+\frac{\mu_{0}}{4 \pi \beta} \mathbf{h} \cdot \nabla \mathbf{h}\right] \\
& -\frac{\mu_{0}}{4 \pi \beta} \nabla \mathbf{h}: \nabla \mathbf{h}
\end{aligned}
$$

where the double contraction is defined as

$$
\nabla \mathbf{u}: \nabla \mathbf{u}=\sum_{i, j=1}^{3}\left(\frac{\partial u_{i}}{\partial x_{j}}\right)^{2}
$$

If the initial data are regular and compactly supported, then Gauss' theorem turns identity (115) into the global energy law

$$
\frac{d}{d t}[K(t)+W(t)+M(t)]=-X_{m}(t)
$$


where

$$
K(t)=\frac{\rho}{2} \int_{\mathbb{R}^{3}}\left|\frac{\partial \mathbf{u}(\mathbf{x}, t)}{\partial t}\right|^{2} d v(\mathbf{x})
$$

denotes the kinetic energy,

$$
W(t)=\frac{1}{2} \int_{\mathbb{R}^{3}}\left[\mu(\nabla \mathbf{u}(\mathbf{x}, t)):(\nabla \mathbf{u}(\mathbf{x}, t))+(\lambda+\mu)|\nabla \cdot \mathbf{u}(\mathbf{x}, t)|^{2}\right] d v(\mathbf{x})
$$

denotes the strain energy,

$$
M(t)=\frac{\mu_{0}}{8 \pi} \int_{\mathbb{R}^{3}}|\mathbf{h}(\mathbf{x}, t)|^{2} d v(\mathbf{x})
$$

denotes the magnetic energy of the disturbance and, finally,

$$
X_{m}(t)=\frac{\mu_{0}}{4 \pi \beta} \int_{\mathbf{R}^{3}}(\nabla \mathbf{h}(\mathbf{x}, t)):(\nabla \mathbf{h}(\mathbf{x}, t)) d v(\mathbf{x})
$$

specifies the magnetic dissipation function. The energy balance law (115) implies that the total (kinetic, plus strain, plus magnetic) energy, is dissipated in time at a rate proportional to the $L^{2}$ norm of the gradient of the magnetic field.

By virtue of Parseval's theorem the energy integrals can be expressed via the Fourier spectral densities of the displacement and the magnetic fields as follows:

$$
\begin{aligned}
K(t) & =\frac{\rho}{2} \int_{\mathbb{R}^{3}}\left|\frac{\partial \hat{\mathbf{u}}(\boldsymbol{\xi}, t)}{\partial t}\right|^{2} d v(\boldsymbol{\xi}), \\
W(t) & =\frac{\rho}{2} \int_{\mathbb{R}^{3}}\left[\nu_{s}^{2}|\hat{\mathbf{u}}(\boldsymbol{\xi}, t)|^{2}+\left(\nu_{p}^{2}-\nu_{s}^{2}\right)|\mathbf{a} \cdot \hat{\mathbf{u}}(\boldsymbol{\xi}, t)|^{2}\right] r^{2} d v(\boldsymbol{\xi}), \\
M(t) & =\frac{\mu_{0}}{8 \pi} \int_{\mathbb{R}^{3}}|\hat{\mathbf{h}}(\boldsymbol{\xi}, t)|^{2} d v(\boldsymbol{\xi}),
\end{aligned}
$$

and

$$
X_{m}(t)=\frac{\mu_{0}}{4 \pi \beta} \int_{\mathbb{R}^{3}}|\hat{\mathbf{h}}(\boldsymbol{\xi}, t)|^{2} r^{2} d v(\boldsymbol{\xi})
$$

We next substitute the canonical forms (107) and (112) into the expressions (122)-(125) for the energies obtaining a large number of integrals of the form

$$
I_{i j}(t)=\int_{\mathbb{R}^{3}} f(\boldsymbol{\xi}) e^{\left(\tau_{i}(\boldsymbol{\xi})+\tau_{j}(\boldsymbol{\xi})\right) t} d v(\boldsymbol{\xi})
$$

for $i, j=1, \ldots, 9$. With the use of the results in Sec. 4 all these integrals are asymptotically analyzed in exactly the same way as the corresponding energy integrals in the thermoelastic case [10] and the asymptotic forms of the energy are obtained. These form an extremely long and tedious task which we avoid repeating here. Taking into consideration symmetries as well as complex conjugation, which does not alter the time 
behaviour of the energy integrals, we still need to find the asymptotic form of 13 integrals for each one of the four energy forms, $K, W, M$, and $X_{m}$.

The final results show that, for smooth enough Cauchy data, the energies satisfy the relations

$$
\begin{aligned}
& K(t)=O\left(t^{-\left(m+\frac{3}{2}\right)}\right), \\
& W(t)=O\left(t^{-\left(m+\frac{3}{2}\right)}\right), \\
& M(t)=O\left(t^{-\left(m+\frac{3}{2}\right)}\right)
\end{aligned}
$$

(see [10], relations (8.62)-(8.64)) as $t \rightarrow \infty$, while the dissipation function decays as $t^{-(m+5 / 2)}$ (see [10], relation (8.79)). The nonnegative integer $m$ is defined as

$$
m=\min \left\{v_{0}+1, v_{1}, v_{h}\right\}
$$

where $v_{0}, v_{1}$, and $v_{h}$ stand for the order of the lowest nonvanishing moment of the initial displacement, the initial velocity, and the initial magnetic field, respectively.

\section{REFERENCES}

[1] A. Banos, Jr., Normal modes characterizing magnetoelastic plane waves, Phys. Rev. 104, 300-305 (1956)

[2] J. Bazer, Geometrical magnetoelasticity, Geophys. J. R. Astr. Soc. 25, 207-237 (1971)

[3] C. M. Bender and S. A. Orszag, Advanced Mathematical Methods for Scientists and Engineers, McGraw-Hill, Singapore, 1984

[4] P. Boulanger, Inhomogeneous magnetoelastic plane waves, Elastic Wave Propagation, Proc. Second I.U.T.A.M.-I.U.P.A.P. Symp. (M. F. McCarthy and M. A. Hayes, eds.), Galway, 1989, pp. 601-606

[5] L. Brand, Vector and Tensor Analysis, John Wiley, New York, 1947

[6] P. Chadwick, Elastic Wave Propagation in a Magnetic Field, 9th Internat. Congr. Appl. Mech., Brussels, Proc. III, 1957, pp. 143-153

[7] S. Chander, Phase velocity and energy loss in magneto-thermo-elastic plane waves, Internat. J. Engrg. Sci. 6, 409*424 (1968)

[8] G. Dassios, Equipartition of energy in elastic wave propagation, Mech. Res. Comm. 6, 45-50 (1979)

[9] G. Dassios, Energy theorems for magnetoelastic waves in a perfectly conducting medium, Quart. Appl. Math. 39, 479-490 (1982)

[10] G. Dassios and M. Grillakis, Dissipation rates and partition of energy in thermoelasticity, Arch. Rational Mech. Anal. 87, 49-91 (1984)

[11] J. W. Dunkin and A. C. Eringen, On the propagation of waves in an electromagnetic elastic solid, Internat. J. Engrg. Sci. 1, 461-495 (1963)

[12] A. C. Eringen and G. A. Maugin, Electrodynamics of Continua I, II, Springer-Verlag, New York, 1989

[13] M. Hermite, Sur la Résolution de l'Équation du Cinquieme Degré, Comptes Rendus 46, 508-515 (1858)

[14] A. B. Jerrard, On Certain Transformations Connected with the Finite Solution of Equations of the Fifth Degree, Philos. Mag. 7, 202-203 (1835)

[15] F. John, Partial Differential Equations, Springer-Verlag, New York, 1982

[16] S. Kaliski, Vibrations and Waves. Part A: Vibrations. Part B: Waves, Polish Scientific Publishers, Warsaw, 1992

[17] V. I. Keilis-Borok and A. S. Munin, Magnetoelastic Waves and the Boundary of the Earth's Core, Izv. Geophys. Ser., 1959, pp. 1529-1541; English translation, 1960, pp. 1089-1095

[18] L. Knopoff, The interaction between elastic wave motions and a magnetic field in electrical conductors, J.Geophys. Res. 60, 441-456 (1955) 
[19] F. E. M. Lilley and D. E. Smylie, Elastic wave motion and a nonuniform magnetic field in electrical conductors, J. Geophys. Res. 73, 6527-6533 (1968)

[20] F. C. Moon, Magneto-Solid Mechanics, John Wiley, New York, 1984

[21] W. Nowacki, Dynamic Problems in Thermoelasticity, Noordhoff, Warsaw, 1975

$[22]$ G. Paria, Magneto-elasticity and magneto-thermo-elasticity, Adv. Appl. Mech. 10, 78-112 (1967)

[23] A. J. Willson, The propagation of magneto-thermo-elastic plane waves, Proc. Cambridge Philos. Soc. 59, 483-488 (1963) 